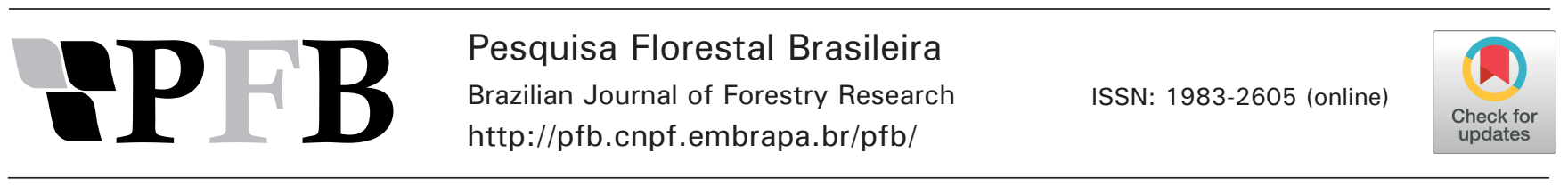

\title{
Comportamento térmico e emissão de gases condensáveis e não condensáveis no processo de carbonização da madeira
}

\begin{abstract}
Wagner Davel Canal1*, Ana Márcia Macedo Ladeira Carvalho ${ }^{1}$, Aylson Costa Oliveira², Mateus Alves de Magalhães ${ }^{1}$,
\end{abstract} Welliton Lelis Cândido ${ }^{1}$, Lucas de Freitas Fialho ${ }^{1}$

1 Universidade Federal de Viçosa, Av. Peter Henry Rolfs, s/n, CEP 36570-900, Viçosa, MG, Brasil

${ }^{2}$ Universidade Federal de Mato Grosso, Av. Fernando Corrêa da Costa, 2367, CEP 78060-900, Cuiabá, MT, Brasil

"Autor correspondente:

wagner.d.canal@gmail.com

Termos para indexação:

Degradação térmica

Carvão vegetal

Eucalyptus

Index terms:

Thermal degradation

Charcoal

Eucalyptus

Histórico do artigo:

Recebido em 16/10/2015

Aprovado em 15/08/2016

Publicado em 30/09/2016

doi: 10.4336/2016.pfb.36.87.1083
Resumo - O objetivo do presente estudo foi avaliar o comportamento térmico e a emissão de gases durante o processo de produção de carvão vegetal a partir de madeira de Eucalyptus spp., com aproximadamente 7 anos de idade. As amostras foram procedentes de um teste clonal de uma empresa florestal, localizada em Divinésia, MG. Realizou-se análise termogravimétrica da madeira (TG/DTG) e carbonizações em mufla, para se determinar o comportamento médio das emissões de gases condensáveis e não condensáveis. A degradação térmica da madeira seguiu comportamento inverso às emissões de dióxido de carbono $\left(\mathrm{CO}_{2}\right)$, monóxido de carbono $(\mathrm{CO})$, metano $\left(\mathrm{CH}_{4}\right)$, hidrogênio $\left(\mathrm{H}_{2}\right)$ e de líquido condensável, passando por um ponto mínimo de degradação e máximo de emissões em temperaturas próximas de $300^{\circ} \mathrm{C}$ e $350^{\circ} \mathrm{C}$, respectivamente. Durante o processo de carbonização foram observados três comportamentos distintos: secagem da madeira até temperaturas próximas a $200^{\circ} \mathrm{C}$; picos máximos de emissão dos gases $\mathrm{CO}_{2}, \mathrm{CO}$ e licor pirolenhoso na faixa de temperatura que corresponde a $300{ }^{\circ} \mathrm{C} \mathrm{e}$ $450{ }^{\circ} \mathrm{C}$; e incrementos dos gases $\mathrm{CH}_{4}$ e $\mathrm{H}_{2}$ de acordo com aumentos em temperatura a partir de $300^{\circ} \mathrm{C}$.

\section{Thermal behavior and emission of condensable and non- condensable gases in the process of wood carbonization}

\begin{abstract}
The main objective of this study was to evaluate wood thermal degradation and the release of condensable and non-condensable gases during charcoal production from Eucalyptus spp. with approximately seven years old. The wood samples were from a clonal test of a forest company from Divinésia, Minas Gerais State, Brazil. Thermogravimetric analysis (TGA/DTA) and carbonizations in muffle were carried out to determine the wood thermal performance and emission factor of non-condensable and condensable gases. Wood thermal degradation presented opposite performance of carbon dioxide $\left(\mathrm{CO}_{2}\right)$, carbon monoxide $(\mathrm{CO})$, methane $\left(\mathrm{CH}_{4}\right)$, hydrogen $\left(\mathrm{H}_{2}\right)$ and condensable gases, presenting a minimum of degradation point and maximum emission at temperatures of $300{ }^{\circ} \mathrm{C}$ and $350{ }^{\circ} \mathrm{C}$, respectively. During carbonization process it was observed three distinct behaviors: drying the wood in temperatures near $200{ }^{\circ} \mathrm{C}$; maximum peak emission of $\mathrm{CO}_{2}, \mathrm{CO}$ and pyrolignous in temperature range corresponding to $300{ }^{\circ} \mathrm{C}$ and $450{ }^{\circ} \mathrm{C}$; and $\mathrm{CH}_{4}$ and $\mathrm{H}_{2}$ gases increases according to temperature increases from $300^{\circ} \mathrm{C}$.
\end{abstract}

\section{Introdução}

O processo de carbonização da madeira objetiva a concentração de carbono no volume disponível, com a eliminação dos elementos voláteis, visando não somente sua transformação em carvão vegetal como também seu alto desempenho e eficiência energética. Segundo Carneiro et al. (2013b), esse processo consiste no aquecimento da madeira a temperaturas entre $350{ }^{\circ} \mathrm{C}$ e $500{ }^{\circ} \mathrm{C}$ com ausência ou presença controlada de oxigênio, promovendo modificações em seus componentes. Essa técnica está intimamente relacionada 
à composição química dos três principais componentes da madeira: celulose, hemiceluloses e lignina, além de sofrer influência das características físicas e anatômicas, que garantem grande parte da resistência térmica da madeira (Oliveira et al., 2010).

É possível obter diferentes faixas de degradação pelo processo de carbonização, uma vez que os constituintes químicos da madeira possuem diversas ligações e inúmeros grupamentos funcionais, resultando, consequentemente, em distintos comportamentos térmicos de acordo com os incrementos em temperatura (Yang et al., 2007). Desse modo, a interpretação das etapas das emissões gasosas torna-se de grande importância a fim de mitigar os efeitos adversos dessa produção sobre a qualidade ambiental.

De modo geral, a carbonização da madeira determina perdas de frações na forma de vapor de água, alcatrões, metano, monóxido de carbono, entre outros compostos, uma vez que a ação do calor tem como intuito eliminar a maior parte dos componentes voláteis da madeira, as quais podem provocar sérios problemas ambientais (Andrade \& Carvalho, 1998; Klose et al., 2000; Carneiro et al., 2013a).

No Brasil, país com grande potencial florestal e único a produzir carvão vegetal como alternativa considerada ecologicamente viável para a produção energética, observou-se produção de carvão vegetal com valores totais de 5,6 milhões de $\mathrm{t}(\mathrm{M} \mathrm{t})$ em 2013, com valores estimados entre $8 \mathrm{M}$ t e $11 \mathrm{M}$ t para 2020 (Brasil, 2015). Desse modo, considera-se de extrema importância entender o processo de carbonização da madeira a fim de mapear as emissões gasosas oriundas desse processo, tendo como intuito buscar soluções para a sua redução, seja com a utilização de um queimador ou com a recuperação desses gases.

Diante de tal contexto, o presente trabalho teve por objetivo descrever as faixas de degradação térmica e a emissão de gases condensáveis e não condensáveis durante o processo de produção de carvão vegetal, além de apresentar os fenômenos termoquímicos que influenciam nesse processo, contribuindo para estudos futuros que busquem minimizar os efeitos adversos do processo de carbonização.

\section{Material e métodos}

Utilizou-se madeira de Eucalyptus spp., com aproximadamente 7 anos de idade, proveniente de um teste clonal localizado na Fazenda Guaxupé, situada no município de Divinésia, $\mathrm{MG}\left(20,974^{\circ} \mathrm{S}\right.$ e $\left.43,088^{\circ} \mathrm{W}\right)$.

\section{Densidade básica da madeira}

Retiraram-se corpos de prova, na forma de cunhas opostas, de discos com aproximadamente $5 \mathrm{~cm}$ de espessura a diferentes alturas do fuste. A determinação da densidade básica da madeira foi realizada por meio da imersão em água, de acordo com a norma $\mathrm{ABNT}$ NBR 11941 (Associação Brasileira de Normas Técnicas, 2003).

\section{Poder calorífico superior da madeira (PCS)}

O poder calorífico superior da madeira foi determinado de acordo com a metodologia descrita pela norma ABNT NBR 8633 (Associação Brasileira de Normas Técnicas, 1984), em duplicatas, utilizando-se uma bomba calorimétrica adiabática IKA300.

\section{Composição química estrutural}

Os teores de extrativos da madeira foram determinados em duplicatas, de acordo com a norma TAPPI 204 OM88 (Technical Association of the Pulp and Paper Industry, 2001), utilizando-se o método de determinação de extrativos totais, substituindo-se o etanol/benzeno por etanol/tolueno.

Os teores de lignina insolúvel foram determinados em duplicatas, pelo método Klason, modificado de acordo com o procedimento proposto por Gomide \& Demuner (1986). O teor de lignina solúvel foi determinado por espectrometria, conforme Goldschimid (1971), a partir da diluição do filtrado proveniente do procedimento para obtenção do teor de lignina insolúvel. O teor de lignina total foi obtido por meio da soma dos teores de lignina solúvel e insolúvel. O teor de holoceluloses foi determinado por diferença, subtraindo-se de 100 os teores de lignina total e extrativos.

\section{Composição química elementar}

A determinação da composição elementar da madeira foi realizada em duplicatas, conforme metodologia descrita por Paula et al. (2011). Utilizou-se massa equivalente a $2,0 \mathrm{mg}( \pm 0,5)$ de serragem seca à temperatura de $105 \pm 2{ }^{\circ} \mathrm{C}$, previamente selecionada em peneiras sobrepostas com malhas de 200 e 270 mesh, sendo utilizada a fração retida nessa última. Em seguida, as amostras foram depositadas no carrossel do equipamento da marca Elementar, modelo Vario Micro Cube CHNS-O, sendo realizada a análise de uma amostra por vez. Os gases necessários para a operação 
foram o hélio, que é o gás de arraste, e o oxigênio, gás de ignição. A temperatura do tubo de combustão, localizado no interior do equipamento no momento da queda da amostra do carrossel, foi de $1.150{ }^{\circ} \mathrm{C}$.

Após a combustão, os gases foram transportados por arraste para o tubo de redução e seguiram para a coluna de detecção. Os elementos químicos (carbono, nitrogênio, hidrogênio e enxofre) foram identificados por meio de um detector de termocondutividade, onde cada elemento tem interação e pico específicos. O teor de oxigênio foi determinado pelo somatório dos teores de C, N, H e S decrescido de 100.

\section{Análises termogravimétricas da madeira}

Para a análise termogravimétrica da madeira utilizou-se o aparelho DTG-60H, Shimadzu. As análises foram realizadas sob atmosfera de gás nitrogênio a uma vazão constante de $50 \mathrm{~mL} \mathrm{~min}^{-1}$, utilizando-se aproximadamente $2 \mathrm{mg}$ de serragem selecionada em peneiras sobrepostas de malha de 200 e 270 mesh, sendo a fração utilizada aquela retida nesta última. As curvas termogravimétricas foram obtidas a partir de $50^{\circ} \mathrm{C}$ até a temperatura máxima de $450^{\circ} \mathrm{C}$, com taxa de aquecimento de $10^{\circ} \mathrm{C} \mathrm{m^{-1 }}$.

A curva termogravimétrica (TG) foi obtida para avaliar a perda de massa em função da temperatura e a curva da derivada primeira (DTG), relacionando a degradação térmica em função do tempo.

A partir das curvas TG, procederam-se os cálculos de perda de massa nos seguintes intervalos de temperatura: $100-150{ }^{\circ} \mathrm{C}, 150-200{ }^{\circ} \mathrm{C}, 200-250{ }^{\circ} \mathrm{C}$, $250-300{ }^{\circ} \mathrm{C}, 300-350{ }^{\circ} \mathrm{C}, 350-400{ }^{\circ} \mathrm{C}, 400-450{ }^{\circ} \mathrm{C}$. Por fim, calculou-se a massa residual na temperatura de $450{ }^{\circ} \mathrm{C}$.

\section{Preparação das amostras para carbonização}

A madeira foi seccionada em dimensões de $2 \mathrm{~cm} \mathrm{x}$ $2 \mathrm{~cm}$ x $5 \mathrm{~cm}$ e seca em estufa de circulação de ar forçado $\left(103 \pm 2{ }^{\circ} \mathrm{C}\right)$, para garantir massa constante e obtê-la absolutamente seca.

Para cada carbonização utilizou-se massa de madeira média de $281 \mathrm{~g}$. As carbonizações foram feitas em forno tipo mufla, sob uma taxa média de aquecimento de 1,67 ${ }^{\circ} \mathrm{C} \mathrm{min}{ }^{-1}$, com incrementos de $50{ }^{\circ} \mathrm{C}$ a cada $30 \mathrm{~min}$. A temperatura inicial foi de $100{ }^{\circ} \mathrm{C}$ e a temperatura final de $450{ }^{\circ} \mathrm{C}$, permanecendo estabilizada nesta última por um período de $90 \mathrm{~min}$, totalizando, portanto, um tempo de carbonização de $5 \mathrm{~h}$.

\section{Coleta de gases não condensáveis}

$\mathrm{Na}$ saída da mufla foi conectado um sistema de recuperação dos gases condensáveis (GC), utilizando um condensador tubular resfriado à água acoplado a um recipiente de coleta.

Os gases não condensáveis (GNC) foram succionados por uma bomba com vazão de coleta ajustada para $1 \mathrm{~L} \mathrm{~min}^{-1}$, sendo conduzidos até o sistema de condicionamento de gases Gasboard 9030 Wuhan CUBIC Optoeletronics Co. Ltda. Antes da injeção no equipamento, os gases passaram por um processo de prélavagem, no qual foram encaminhados primeiramente a um kitassato para condensação do licor pirolenhoso mais denso, seguindo para posterior kitassato contendo água para resfriamento adicional dos gases e mais outros dois frascos lavadores de gás do tipo Dreschel contendo álcool absoluto e algodão envolvidos com gelo e armazenados em isopor. A lavagem foi necessária para evitar que possíveis gotículas de alcatrão que porventura não tivessem condensado no primeiro kitassato viessem a fazê-lo na tubulação do Gasboard, saturando o aparelho. Após esse caminho de pré-lavagem, os GNCs seguiram para o interior do aparelho Gasboard 9030 e foram conduzidos para outra lavagem em água, resfriados a $4{ }^{\circ} \mathrm{C}$ em desumidificador (chiller) e, posteriormente, passados em cilindro contendo carvão ativado e algodão. Em seguida, foi realizada a última limpeza dos gases por meio de filtros de precisão, denominados FIT1 e FIT2, os quais retêm impurezas maiores que $3 \mu \mathrm{m}$ e $1 \mu \mathrm{m}$, respectivamente.

Essa sucção foi realizada em intervalos de temperatura de $50^{\circ} \mathrm{C}$ até a temperatura final de $450{ }^{\circ} \mathrm{C}$, sendo feitas duas coletas para cada intervalo, onde a primeira foi entre o $12^{\circ}$ e o $15^{\circ} \mathrm{min}$ e a segunda entre o $27^{\circ}$ e o $30^{\circ} \mathrm{min}$.

Concomitante a esse sistema de purificação externa e interna ao aparelho de sucção, os gases GNC seguiram até o sistema de análise de gás, denominado Gasboard 3100 Wuhan CUBIC Optoeletronics Co. Ltda. Este aparelho forneceu leituras da composição percentual (base volume) de $\mathrm{CH}_{4}, \mathrm{CO}_{2}, \mathrm{CO}$ e $\mathrm{H}_{2}$, correspondendo a um total de 20 registros para cada carbonização.

A média dos dados de concentração dos principais gases de efeito estufa, como o metano $\left(\mathrm{CH}_{4}\right)$, dióxido de carbono $\left(\mathrm{CO}_{2}\right)$, monóxido de carbono $(\mathrm{CO})$ e hidrogênio $\left(\mathrm{H}_{2}\right)$, foi obtida para as três repetições até a temperatura final de carbonização. Para isso, confeccionaram-se curvas percentuais de liberação de cada componente 
gasoso mediante sua evolução, caracterizando todo o processo em termos de emissão. Além disso, com os dados de concentração de cada gás e da massa dos gases não condensáveis, foi possível mensurar a massa total (g) de cada componente do gás não condensável emitida até $450{ }^{\circ} \mathrm{C}$, utilizando o balanço de massa descrito na Tabela 1, conforme Taccini (2010).

Tabela 1. Balanço de massa dos gases não condensáveis.

\begin{tabular}{cl}
\hline $\begin{array}{c}\text { Massa específica } \\
\text { gases não } \\
\text { condensáveis }\end{array}$ & \multicolumn{1}{c}{ Equações } \\
\hline$\left(\boldsymbol{\rho}_{\text {gas }}, \mathbf{e m ~ k g}_{\text {gas }} \mathbf{m}_{\text {gas }}^{-3}\right)$ & $\rho_{\text {gas }}=\left[(44 / 0,224) * \mathrm{X}_{\mathrm{CO} 2}\right]+\left[(28 / 0,224) * \mathrm{X}_{\mathrm{CO}}\right]+\left[(2 / 0,224) * \mathrm{X}_{\mathrm{H} 2}\right]+\left[(16 / 0,224) * \mathrm{X}_{\mathrm{CH} 4}\right]$ \\
\hline & $\mathrm{M}_{\mathrm{CO} 2}=\left[(44 / 0,224) * \mathrm{X}_{\mathrm{CO} 2} / \rho_{\mathrm{gas}}\right] * \mathrm{M}_{\mathrm{GNC}}$ \\
& $\left.\mathrm{M}_{\mathrm{CO}}=[28 / 0,224) * \mathrm{X}_{\mathrm{CO}} / \rho_{\mathrm{gas}}\right] \mathrm{M}_{\mathrm{GNC}}$ \\
& $\mathrm{M}_{\mathrm{H} 2}=\left[(2 / 0,224) * \mathrm{X}_{\mathrm{H} 2} / \rho_{\mathrm{gas}}\right] * \mathrm{M}_{\mathrm{GNC}}$ \\
& $\mathrm{M}_{\mathrm{CH} 4}=\left[(16 / 0,224) * \mathrm{X}_{\mathrm{CH} 4} / \rho_{\mathrm{as}}\right] * \mathrm{M}_{\mathrm{GNC}}$ \\
&
\end{tabular}

Em que: $\mathrm{X}_{\mathrm{CO} 2}, \mathrm{X}_{\mathrm{CO}}, \mathrm{X}_{\mathrm{H} 2}, \mathrm{X}_{\mathrm{CH} 4}$ (porcentagem mássica média de cada gás); $\mathrm{M}_{\mathrm{CO} 2} ; \mathrm{M}_{\mathrm{CO}} ; \mathrm{M}_{\mathrm{H} 2} ; \mathrm{M}_{\mathrm{CH} 4}$ (massa de cada gás, em g); $\mathrm{M}_{\mathrm{GNC}}$ (massa dos gases não condensáveis).

Os valores obtidos de $\mathrm{M}_{\mathrm{CO} 2}, \mathrm{M}_{\mathrm{CO}}, \mathrm{M}_{\mathrm{H} 2}$ e $\mathrm{M}_{\mathrm{CH} 4}$ foram transformados em fator de emissão $\left(\mathrm{Kg}_{\text {gás }} \cdot \mathrm{t}^{-1}{ }_{\text {madeira }}\right)$ com base na massa de madeira utilizada em cada carbonização, extrapolada para $1.000 \mathrm{~kg}$, sendo descrito na Equação 1.

$f=M_{y} * \frac{1.000}{M m a d s i}$

Em que: $M_{\gamma}=$ massa de cada gás $\gamma$, em g; Mmads $i=$ massa de madeira seca correspondente a cada carbonização $i$, em g.

\section{Sistema de coleta de gases condensáveis}

Para a recuperação dos gases condensáveis utilizouse somente um condensador tubular resfriado, trocador de calor gás/água, acoplado a um recipiente de coleta (kitassato).

Nessa medição da massa de gás emitido, foram realizadas três novas carbonizações com mesma madeira, massa média e marcha de carbonização, substituindo-se o sistema de tratamento e análise dos gases não condensáveis por um sistema de sequestro de gases condensáveis. A quantificação acumulada da massa de gases condensáveis emitida foi realizada com a utilização de dois kitassatos tarados inicialmente.

\section{Análise descritiva dos dados}

Os dados da análise com um tratamento padrão (madeira totalmente seca) e três repetições (carbonizações) foram submetidos à análise descritiva, com manipulação das médias dos dados das propriedades físicas e químicas da madeira e do rendimento dos produtos da carbonização, além de terem sido confeccionados gráficos descritivos do comportamento da carbonização. Para todas essas análises utilizou-se planilha eletrônica.

\section{Resultados e discussão}

\section{Propriedades da madeira}

A densidade básica da madeira foi igual a $0,48 \mathrm{~g} \mathrm{~cm}^{-3}$, valor inferior ao citado por Neves et al. (2011), que encontraram valores próximos a $0,51 \mathrm{~g}$ $\mathrm{cm}^{-3}$ para clones do mesmo gênero desenvolvidos por uma empresa florestal localizada em Minas Gerais. Encontrou-se também poder calorífico superior médio igual a $4.485 \mathrm{kcal} \mathrm{kg}^{-1}$, compatível com os valores encontrados por Brand \& Muñiz (2010) para o mesmo gênero na produção de biomassa para geração de energia. No entanto, esse valor não é considerado como potencial para a produção de carvão vegetal, uma vez que é resultado da matéria-prima que lhe deu origem e do processo de carbonização utilizado (Carneiro et al., 2013b).

A madeira continha 70,5\% de holoceluloses, $26,5 \%$ de lignina e 3,1\% de extrativos. Para a produção de carvão vegetal opta-se por madeiras com baixo teor de celulose e hemiceluloses, uma vez que estes componentes apresentam baixa resistência à degradação térmica e interferem significativamente na emissão de compostos gasosos (Yang et al. 2007; Shen et al., 2010). A lignina apresenta-se como principal parâmetro para escolha de clones com características satisfatórias para produção de carvão vegetal, uma vez que aumentos de seus 
teores para valores próximos de $32 \%$ podem promover significativos ganhos de conversão de madeira em carvão vegetal (Brasil, 2015). Além disso, elevados teores de extrativos também não interferem no ganho em massa de carvão vegetal, uma vez que a maioria destes compostos degrada-se entre $200^{\circ} \mathrm{C}$ e $500^{\circ} \mathrm{C}$, devido à sua natureza química (Grønli et al., 2002; Shebani et al., 2008).

\section{Degradação térmica da madeira}

Na Figura 1 é apresentada a curva termogravimétrica (TGA) da madeira e sua derivada (DTG).

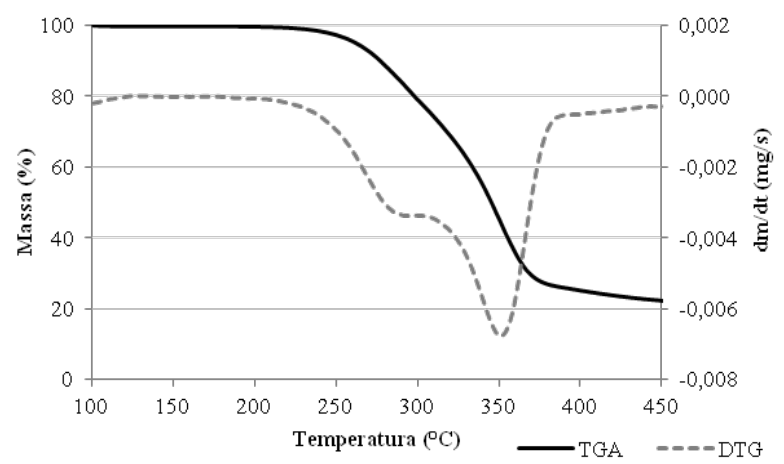

Figura 1. Curvas termogravimétrica da madeira de Eucalyptus spp. (TGA) e sua derivada (DTG).

Por se tratar de um material completamente seco em estufa, não se observa claramente o primeiro pico de perda de massa da madeira com relação ao incremento em temperatura (Figura 1), a qual se deve à fase de secagem da madeira, que ocorre até $200{ }^{\circ} \mathrm{C}$ (Carneiro et al., 2013b).

Após essa primeira faixa de aquecimento, observase um pico de perda de massa entre as temperaturas de $200^{\circ} \mathrm{C}$ e $290^{\circ} \mathrm{C}$, com perda de $16,4 \%$ em massa devido à degradação das hemiceluloses (Figura 1). No intervalo entre $310^{\circ} \mathrm{C}$ e $400{ }^{\circ} \mathrm{C}$ observa-se uma queda de $48,7 \%$ na curva TGA, em consequência da degradação térmica da celulose, que segue até temperaturas próximas do fim do processo (Shen et al., 2010). Entre as temperaturas de $400{ }^{\circ} \mathrm{C}$ e $450{ }^{\circ} \mathrm{C}$ observou-se uma estabilização quanto à perda de massa do material, uma vez que a resistência térmica da lignina facilita a fixação de carbono no produto formado (Yang et al., 2007).

Pode-se observar também que a degradação segue para uma pequena estabilização entre as temperaturas de $290{ }^{\circ} \mathrm{C}$ e $310^{\circ} \mathrm{C}$ (Figura 1), o qual caminha para um estado de transição da degradação das hemiceluloses e celulose.
Portanto, grandes diferenças de resistência térmica podem ser observadas para os três constituintes da madeira: hemiceluloses, celulose e lignina. Cabe destacar ainda que a degradação térmica de qualquer biomassa, entre elas a madeira, pode ser considerada como a sobreposição da decomposição destes três componentes, além de sua secagem (Bianchi et al., 2010; Pereira et al., 2013).

\section{Efeito da temperatura de carbonização nas emissões percentuais de gases não condensáveis e licor pirolenhoso}

$\mathrm{Na}$ Figura 2 é apresentada a curva percentual de emissão de metano $\left(\mathrm{CH}_{4}\right)$, dióxido de carbono $\left(\mathrm{CO}_{2}\right)$, monóxido de carbono $(\mathrm{CO})$, hidrogênio $\left(\mathrm{H}_{2}\right)$ e licor pirolenhoso (LP) em função da temperatura de carbonização.

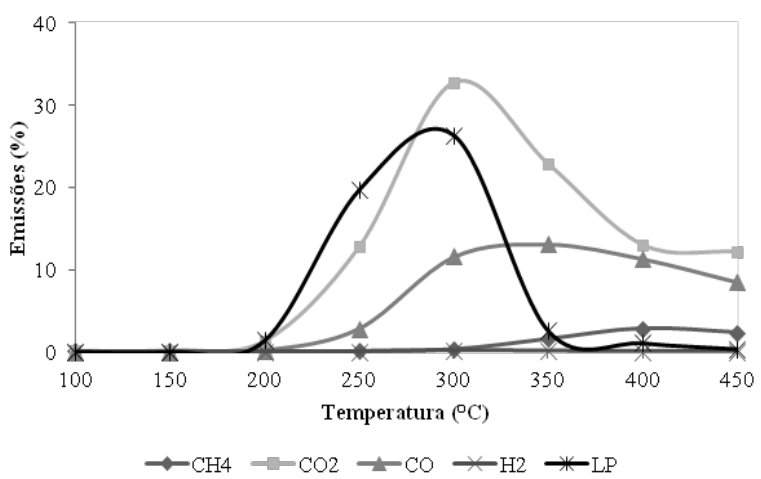

Figura 2. Percentual de emissão de gases condensáveis (LP) e não condensáveis $\left(\mathrm{CH}_{4}, \mathrm{CO}_{2}, \mathrm{CO}, \mathrm{e} \mathrm{H}_{2}\right)$ de madeira de Eucalyptus spp.

Nas temperaturas compreendidas entre $100{ }^{\circ} \mathrm{C}$ e $200{ }^{\circ} \mathrm{C}$ não se observou liberação significativa de gases não condensáveis ou emissão de licor pirolenhoso (LP), devido à madeira ter sido seca anteriormente à carbonização. Nessa faixa de temperatura os constituintes químicos da madeira que possuem baixa resistência térmica, como as hemiceluloses e a celulose, começaram a se degradar, percebendo-se picos emissivos muito baixos, com valores da ordem de $1,3 \% ; 0,20 \%$; $0,077 \%$ e $0,0 \%$ para os gases $\mathrm{CO}_{2}, \mathrm{CO}, \mathrm{CH}_{4}$ e $\mathrm{H}_{2}$, respectivamente. Assim, pode-se considerar que até temperaturas próximas de $200{ }^{\circ} \mathrm{C}$ ocorre secagem da madeira e nos limites dessa temperatura também se constatam perdas de massa e consequente liberação de produtos gasosos. 
Elevação da temperatura a partir de $200{ }^{\circ} \mathrm{C}$ promove aumentos progressivos nas emissões de gases não condensáveis e condensáveis, tendo-se encontrado o pico máximo de emissões gasosas na faixa de temperatura que vai de $300{ }^{\circ} \mathrm{C}$ até $350{ }^{\circ} \mathrm{C}$. Foi observada a formação de $32,69 \%$ de $\mathrm{CO}_{2} ; 11,58 \%$ de $\mathrm{CO} ; 0,14 \%$ de $\mathrm{H}_{2} ; 26,28 \%$ do licor condensável na faixa de temperatura próxima a $300{ }^{\circ} \mathrm{C}$, e $2,81 \% \mathrm{de} \mathrm{CH}_{4}$ na faixa de temperatura próxima a $400{ }^{\circ} \mathrm{C}$.

A eliminação de compostos orgânicos entre as temperaturas de $200^{\circ} \mathrm{C} \mathrm{e} 350^{\circ} \mathrm{C}$ provavelmente se deve à quebra das ligações $\mathrm{OH}$ da celulose, hemiceluloses e de grupos metoxila dos anéis aromáticos que compõem a lignina (Yang et al, 2007; Shen et al. 2010). Suarez et al. (2007) afirmaram que nessa faixa de temperatura ocorre a intensificação da quebra das moléculas dos constituintes químicos da madeira, levando à formação de uma mistura de hidrocarbonetos e compostos oxigenados, lineares ou cíclicos, tais como alcanos, alquenos, cetonas, ácidos carboxílicos e aldeídos, além de monóxido e dióxido de carbono e água.

Por fim, entre as temperaturas de $350{ }^{\circ} \mathrm{C}$ e $450{ }^{\circ} \mathrm{C}$ não foi observada produção significativa de líquido condensável, porém as emissões dos gases $\mathrm{CO}_{2}, \mathrm{CO}, \mathrm{H}_{2}$ e $\mathrm{CH}_{4}$ seguiu em incrementos consideráveis, assim como encontrado por Yang et al. (2007) e Shen et al. (2010), observando-se sobretudo o primeiro grande pico de $\mathrm{CH}_{4}\left(2,81 \%\right.$ para a temperatura de $\left.400^{\circ} \mathrm{C}\right)$. Além disso, próximo a temperatura de $450{ }^{\circ} \mathrm{C}$ tem-se a formação de carvão, que é resultante da fixação de carbono ao material resultante, havendo pouca ou nenhuma contribuição das hemiceluloses e celulose, devendo ser encerrado visando rendimento significativo e qualidade do carvão (Oliveira et al. 2010; Pereira et al., 2013).

Cabe destacar que para a faixa de temperatura entre $300{ }^{\circ} \mathrm{C}$ e $400{ }^{\circ} \mathrm{C}$ foi observada uma fumaça de coloração amarelada, indicando a formação de novos compostos e abrindo novos caminhos de estudos para esse comportamento.

\section{Conclusões}

Picos específicos de emissão de gases condensáveis e não condensáveis podem ser observados em faixas específicas de temperatura durante o processo de carbonização, passando por um ponto de máximo entre as temperaturas de $300{ }^{\circ} \mathrm{C}$ e $350{ }^{\circ} \mathrm{C}$, a qual se relaciona com o ponto de degradação máxima das hemiceluloses e celulose.
Durante o processo de carbonização são observadas três fases: aquecimento da madeira sem perda de massa e emissões significativas até temperaturas próximas a $200{ }^{\circ} \mathrm{C}$; picos máximos de degradação e emissão dos gases $\mathrm{CO}_{2}, \mathrm{CO}$ e licor pirolenhoso na faixa de temperatura que corresponde a $300{ }^{\circ} \mathrm{C}$ e $450{ }^{\circ} \mathrm{C}$; e incrementos na produção dos gases $\mathrm{CH}_{4}$ e $\mathrm{H}_{2}$ de acordo com aumentos em temperatura a partir de $300^{\circ} \mathrm{C}$.

\section{Agradecimentos}

Os autores agradecem à Fundação de Amparo à Pesquisa do Estado de Minas Gerais - FAPEMIG, ao Conselho Nacional de Pesquisa e Desenvolvimento - CNPq e à Secretaria de Estado de Ciência, Tecnologia e Ensino Superior - SECTES pelo apoio financeiro; à Universidade Federal de Lavras pela realização das análises térmicas da madeira; e à Embrapa Florestas pelo apoio dado para realização desse trabalho.

\section{Referências}

Andrade, A. M. \& Carvalho, L. M. Potencialidades energéticas de oito espécies florestais do Estado do Rio de Janeiro. Floresta e Ambiente, v. 5, n.1, p. 24-42, 1998.

Associação Brasileira de Normas Técnicas. NBR 8633: carvão vegetal: determinação do poder calorífico. Rio de Janeiro, 1984. 13 p.

Associação Brasileira de Normas Técnicas. NBR 11941: madeira: determinação da densidade básica. Rio de Janeiro, 2003. 6 p.

Bianchi, O. et al. Avaliação da degradação não-isotérmica da madeira através de termogravimetria - TGA. Polímeros, v. 20, n. 5, p. $395-$ 400, 2010. DOI: 10.1590/S0104-14282010005000060.

Brand, M. A. \& Muñiz, G. I. B. Influência da época de colheita da biomassa florestal sobre sua qualidade para a geração de energia. Scientia Forestalis, v. 38, n. 88, p. 619-628, 2010.

Brasil. Ministério do Desenvolvimento da Indústria e Comércio Exterior. Centro de Gestão e Estudos Estratégicos. Modernização da produção de carvão vegetal: subsídios para revisão do plano siderurgia. Brasília, DF, 2015. 154 p. Disponível em: $<$ https://cgee.org.br/documents/10182/734063/Carvao_Vegetal_ WEB_02102015_10225.PDF>. Acesso em: 19 dez. 2015.

Carneiro, A. C. O. et al. Estudo da decomposição térmica da madeira de oito espécies da Região do Seridó, Rio Grande do Norte. Revista Árvore, v. 37, n. 6, p. 1153-1163, 2013a. DOI: 10.1590/S010067622013000600017.

Carneiro, A. C. O. et al. Pirólise lenta da madeira para produção de carvão vegetal. In: Santos, F. et al. (Org.). Bioenerga \& Biorrefinaria: cana de açúcar \& espécies florestais. Visconde do Rio Branco: Suprema, 2013b. v. 1. p. 429-458. 
Goldschimid, O. Ultraviolet spectra. In: Sarkanen, K. V. \& Ludwing, C. H. (Ed.). Lignins. New York: Wiley Interscience, 1971. p. 241266.

Gomide, J. L. \& Demuner, B. J. Determinação do teor de lignina em material lenhoso: método Klason modificado. O Papel, v. 47, n. 8, p. 36-38, 1986.

Grønli, M. G. et al. Thermogravimetric analysis and devolatilization kinetics of wood. Industry Engineering Chemistry Resources, v. 41, n. 17, p. 4201-4208, 2002. DOI: 10.1021/ie0201157.

Klose, W. et al. Pyrolysis and activation of different woods Thermal analysis (TG/EGA) and formal kinetics. In: INTERNATIONAL SYMPOSIUM OF CATALYTIC AND THERMOCHEMICAL CONVERSIONS OF NATURAL ORGANIC POLYMERS, 4., 2000, Krasnoyarsk. Proceedings. [S.1.]: Institut Chimii i Chimiceskoj Technologii, 2000.

Neves, T. A. et al. Avaliação de clones de Eucalyptus em diferentes locais visando à produção de carvão vegetal. Pesquisa Florestal Brasileira, v. 31, n. 68, p. 319-330, 2011.

Oliveira, A. C. et al. Parâmetros de qualidade da madeira e do carvão vegetal de Eucalyptus pellita F. Muell. Scientia Forestalis, v. 38, n. 87, p. 431-439, 2010.

Paula, L. R. et al. Characterization of residues from plant biomass for use in energy generation. Cerne, v. 17, n. 2, p. 237-246, 2011. DOI: $10.1590 / \mathrm{S} 0104-77602011000200012$.
Pereira, B. L. C. et al. Estudo da degradação térmica da madeira de Eucalyptus através de termogravimetria e calorimetria. Revista Árvore, v. 37, n. 3, p. 567-576, 2013. DOI: 10.1590/S010067622013000300020 .

Shebani, A. N. et al. The effect of wood extractives on the termal stability of diferente wood species. Thermochim Acta, v. 471, p. 43-50, 2008. DOI: 10.1016/j.tca.2008.02.020.

Shen, R. et al. The thermal performance of the polysaccharides extracted from hardwood: cellulose and hemicelluloses. Carbohydrate Polymers, v. 82, n. 1, p. 39-45, 2010. DOI: 10.1016/j. carbpol.2010.04.018.

Suarez, P. A. Z. et al. Transformação de triglicerídeos em combustíveis materiais poliméricos e insumos químicos: algumas aplicações da catálise na oleoquímica. Química Nova, v. 30, n. 3, 667-676, 2007.

Taccini, M. M. Estudo das metodologias da convenção-quadro das nações unidas sobre mudanças climáticas, referentes à avaliação de emissões de gases de efeito estufa na produção de carvão vegetal. 2010. 87 f. Dissertação (Mestrado em Ciência Florestal) Escola Superior de Agricultura "Luiz Queiroz", Piracicaba.

Technical Association of the Pulp and Paper Industry. TAPPI test methods T 204 om-88: solvent extractives of wood and pulp. Atlanta, 2001. Cd-Rom

Yang, H. et al. Characteristics of hemicellulose, cellulose and lignin pyrolysis. Fuel, n. 86, p. 1781-1788, 2007. DOI: 10.1016/j. fuel.2006.12.013. 
